\title{
1 A comparison of two methods for estimating measurement 2 repeatability in morphometric studies
}

3 Zachariah Wylde and Russell Bonduriansky

4 Evolution and Ecology Research Centre, School of Biological, Earth and Environmental

5 Sciences, University of New South Wales, Sydney, NSW, Australia.

\section{Summary}

7 1. Measurement repeatability is often reported in morphometric studies as an index of the

8 contribution of measurement error to trait measurements. However, the common method

9 of remeasuring a mounted specimen fails to capture some components of measurement

10 error, and could therefore yield inflated repeatability estimates. Remounting specimens

11 between successive measurements is likely to provide more realistic estimates of

12 repeatability, particularly for small structures that are difficult to measure.

13 2. Using measurements of 22 somatic and genitalic traits of the neriid fly Telostylinus

14 angusticollis, we compared repeatability estimates obtained via remeasuring (where a

15 mounted specimen is measured twice) versus remounting (where a specimen is remounted

16 between measurements). We also asked whether the difference in repeatability estimates

17 obtained via the two methods depends on trait size.

18 3. Repeatability estimates obtained via remounting were lower than estimates obtained via

19 remeasuring for each of the 22 traits, and the difference between estimates obtained via

20 the two methods was generally greater for small structures (genitalic traits) than for large

21 structures (legs, wings).

22 4. Remounting specimens between successive measurements can provide more accurate

23 estimates of measurement repeatability than remeasuring from a single mount, especially

24 for small structures that are difficult to measure.

\section{KEYWORDS}

26 Repeatability, phenotype, allometry, measurement error, precision 


\section{Introduction}

28 Repeatability is estimated from repeated measurements taken on several individuals, and is

29 typically calculated as the intra-class correlation coefficient-i.e., the proportion of total

30 variance that is attributable to individual identity (Lessells \& Boag, 1987) (Sokal \& Rohlf,

31 1995; Stoffel, Nakagawa, \& Schielzeth, 2017). Repeatability is estimated for many different

32 reasons (Wilson, 2018). In morphometric studies, repeatability is useful as a gauge of the

33 contribution of measurement error to trait measurements, and therefore the statistical

34 power of analyses involving those measurements (Bailey \& Byrnes, 1990; Yezerinac,

35 Lougheed, \& Handford, 1992). In principle, if Trait A has greater measurement error than

36 Trait B, Trait A will have lower repeatability than Trait B, and analyses of variation in Trait A

37 will have lower statistical power. From a statistical standpoint, the calculation of

38 repeatability has received considerable attention in the literature (Lessells \& Boag 1987;

39 (Altaye, Donner, \& Klar, 2001; Shoukri \& Donner, 2001; Ghosh \& Das, 2003; Stoffel et al.,

40 2017).

41 However, specimen handling and remeasurement methods can also affect repeatability

42 estimates. For example, a study on the morphometrics of skeletal traits found that, as

43 measuring technique improved through experience, measurement error declined (Yezerinac

44 et al., 1992). The repeatability of trait measurements can depend on both intrinsic (e.g.

45 genetic) and extrinsic (e.g. environmental) factors (Wilson, 2018). The way in which one

46 handles and measures a specimen is likely to be an important, but somewhat overlooked

47 extrinsic factor that also influences repeatability. Guidelines exist on the number of

48 individuals and number of measurements per individual required to obtain precise

49 estimates of repeatability (Wolak, Fairbairn, \& Paulsen, 2012), but guidelines on appropriate

50 specimen handling and remeasurement techniques are currently lacking.

51 To provide useful estimates of the contribution of measurement error to trait

52 measurements, repeatability estimates must capture as many sources of measurement

53 error as possible. In morphometric studies (particularly those involving small specimens,

54 such as insect body parts), measurement error typically reflects how specimens are

55 mounted, and how their dimensions are quantified. A common procedure is to remeasure a

56 single mount (or an image of a single mount). This method has been used widely in studies 
57 by our lab and other groups (Blanckenhorn, Kraushaar, Teuschl, \& Reim, 2004; Hosken,

58 Minder, \& Ward, 2005; Bertin \& Fairbairn, 2007; Bonduriansky, 2007; Cayetano \&

59 Bonduriansky, 2015; Bonduriansky et al., 2015). Many other morphometric studies do not

60 specify how repeatability was estimated, or do not report repeatability at all. However, this

61 method omits sources of measurement error associated with specimen mounting. For

62 example, each time a specimen is mounted its orientation relative to the focal plane of the

63 microscope or camera will be slightly different, resulting in different degrees of parallax

64 error. Soft specimens may also be distorted slightly each time they are handled, and images

65 of specimens mounted in fluid medium (such as glycerol or saline solution) may be

66 shadowed or distorted in various ways by the fluid.

67 Remounting specimens between successive measurement is therefore likely to yield better

68 estimates of measurement repeatability. However, remounting takes time and effort, and it

69 is not clear how substantially estimates of repeatability obtained by remounting would

70 differ from estimates obtained by remeasuring the same mount, or whether the difference

71 between estimates obtained by these methods varies between traits that can be measured

72 with relatively little error (such as large, flat and stiff morphological structures) and traits

73 that are subject to greater measurement error (such as small or soft morphological

74 structures). To address these questions, we compared repeatability estimates obtained via

75 remeasuring and remounting for 22 morphological traits of the neriid fly Telostylinus

76 angusticollis. The traits included small and weakly sclerotized genitalic structures as well as

77 relatively large, strongly sclerotized and flat structures (legs, wings).

\section{Material and methods}

79 STUDY SYSTEM

80 We utilised a morphometric dataset on the neriid fly Telostylinus angusticollis that included

8122 somatic and genitalic traits measured on individuals reared on "rich" and "poor" larval

82 diets (Wylde \& Bonduriansky, submitted). Briefly, eggs collected from stock flies were

83 reared using a nutrient-intermediate larval diet based on (Sentinella, Crean, \&

84 Bonduriansky, 2013), and randomly chosen adults were then paired to create 17 mating

85 pairs. From each pair, 40 eggs were split equally between nutrient-poor and nutrient-rich

86 larval diet treatments (Sentinella et al., 2013). Adults emerging from these larval diets were 
87 frozen for measurement $\sim 24 \mathrm{~h}$ after emergence (i.e., once their exoskeletons had

88 sclerotized fully). Larval diet manipulation influences adult body size and shape in $T$.

89 angusticollis (Bonduriansky, 2007), and therefore increased the range of variation in the

90 sizes of morphological traits examined in this study.

91 We measured six genitalic and 12 somatic traits on each of 93 males ( $n=43$ poor diet, $n=50$

92 rich diet), and four genitalic and 11 somatic traits on each of 96 females ( $n=49$ poor diet, $n$

$93=47$ rich diet). All trait measurements were lengths in mm except for testis area, which was

94 measured in $\mathrm{mm}^{2}$ (see Figure 1, 2 for definitions of trait measurements). Two methods were

95 used to estimate repeatability for each trait. First, each specimen was mounted on the slide

96 and imaged, and then remounted and reimaged; separate measurements were then made

97 from the two images ('remounting' method). Second, each trait was measured twice from a

98 single image ('remeasuring' method). For the remeasuring method, we chose which of the

99 two images to remeasure based on a random sequence of numbers generated from a

100 binomial distribution.

101 SAMPLE PREPARATION

102 For each individual, the head, wings, legs and antennae were separated from the thorax and

103 the genitalia were dissected out. Body parts were laid flat onto 1-1.2 mm microscope slides

$104 \quad\left(\mathrm{ISCO}^{\circledR}\right)$ with an in-built micrometre for measurement calibration. To minimize parallax

105 error, heads were positioned on slides covered with double-sided tape. Genitalic structures

106 were mounted in $7.2 \mathrm{pH}$ Phosphate Buffered Saline (PBS) and covered with $22 \mathrm{~mm}$

107 coverslips. The external genitalia (epandrium), male surstyli (proximal and distal) and

108 internal section of the genitalia (carefully removed as one unit that included the apodeme,

109 aedeagus and processes) were separated from the epandrium and placed under a coverslip.

110 All somatic (both sexes) and male genitalic traits were imaged using a Leica MZ $16 \mathrm{~A}$

111 stereoscope fitted with a Leica MC170 HD camera. Before dissection of spermathecae, the

112 female oviscape was imaged and its length measured. The female reproductive tract with

113 spermathecae was then carefully removed, cleaned and mounted in PBS as described

114 above. Spermathecae were imaged using a Zeiss Axioskop 40 compound microscope fitted

115 with a DinoEyepiece ${ }^{\circledR}$ camera at $20 X$ magnification. 


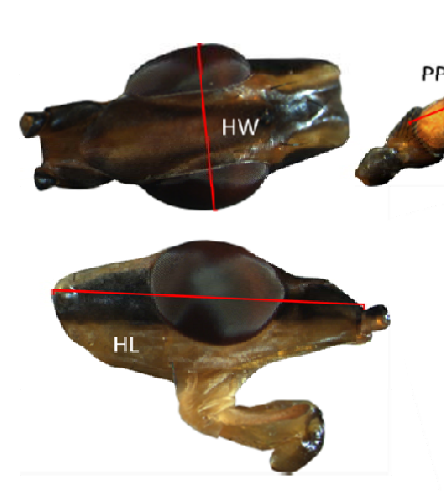

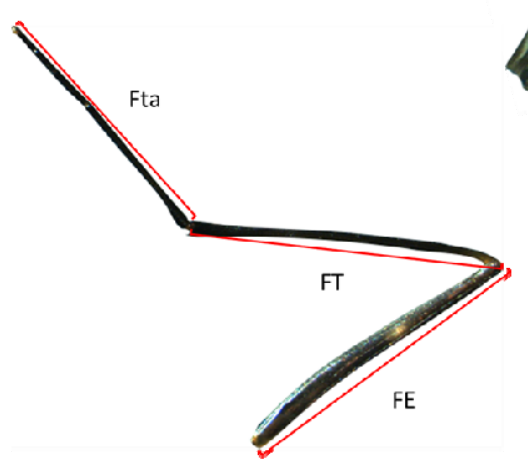

i hind leg

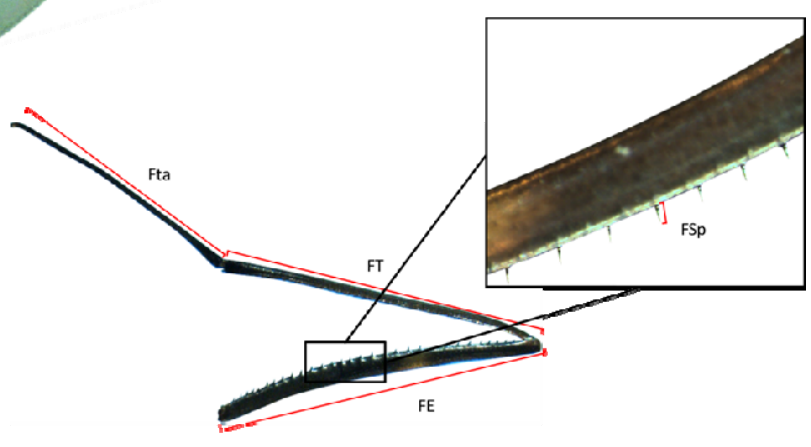

đ front leg

116

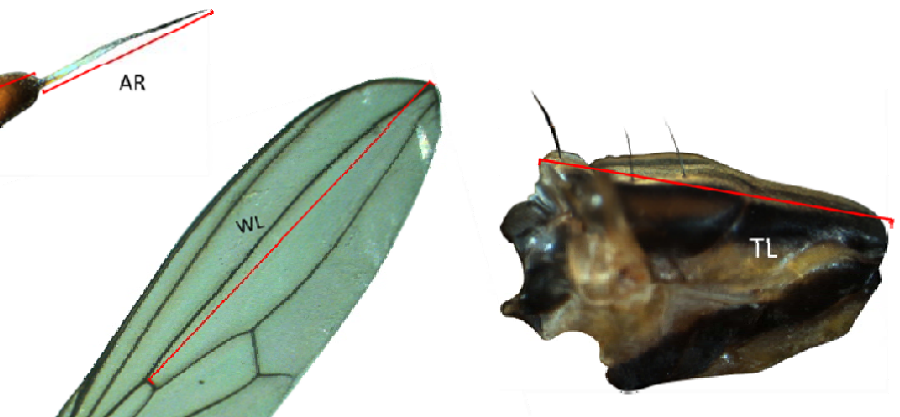

117 Fig. 1. Larger sclerotized body parts of T. angusticollis. Shared traits between male and

118 female include HW (head width), HL (head length), PP (post-pedicel length), AR (arista

119 length), WL (Wing length) and TL (thorax length). In females the hind leg was measured,

120 whereas in males the front leg was measured. Both legs were broken down into

121 measurements of FE (femur length), FSp (femur spine length, males only), FT (tibia length),

122 Fta (tarsus length). Trait images are not to scale. 

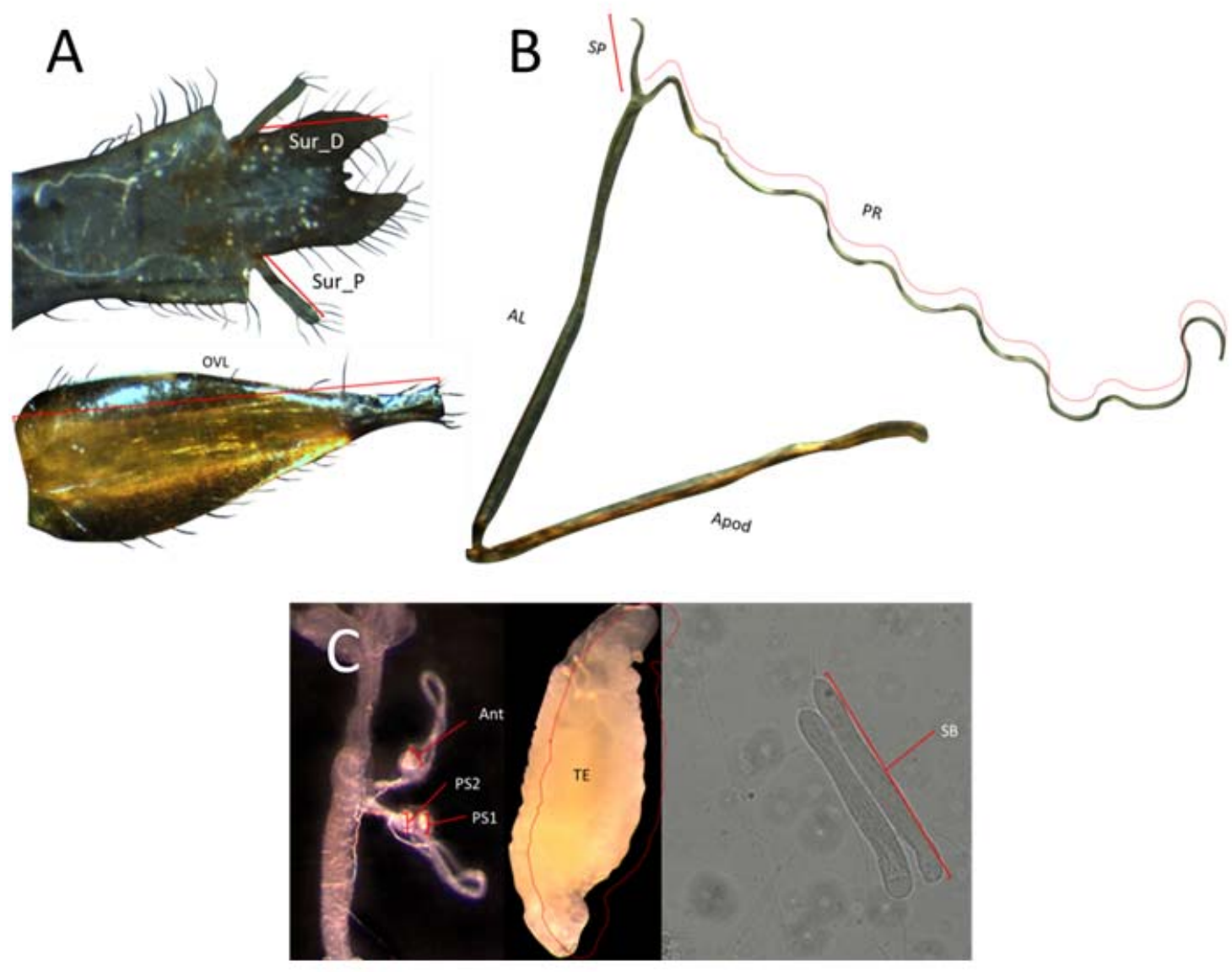

124 Fig. 2. Genitalic and other smaller and unsclerotized traits of T. angusticollis. A: External

125 genitalic traits: male Sur_P (proximal surstyli length) and Sur_D (distal surstyli length);

126 female oviscape (OVL). B: Male genitalic apparatus: Apod (apodeme length), AL (Aedeagus

127 length), SP (short anterior processus length), PR (processus length). C: left, female genitalic

128 apparatus: PS1, PS2 (posterior spermathecae 1 \& 2 width), Ant (anterior spermatheca

129 width); male TE (testis, area $\mathrm{mm}^{2}$ ) and SB (sperm bundle length).

\section{STASTICAL ANALYSIS}

131 All analyses were carried out using R 3.6.2 (R Core Team, 2019). Repeatability was calculated

132 for all traits as the variance among individual trait means (individual-level variance VI) over

133 the sum of individual-level and residual variance VR: $R=V G /(V G+V R)$. We split the data by

134 sex and method of measurement (remeasured or remounted) and fit separate linear mixed

135 models using the packages "ImerTest" (Bates, Maechler, Bolker, \& Walker, 2015;

136 Kuznetsova, Brockhoff, \& Christensen, 2017) and "Ime4" (Bates et al., 2015) where trait size 
137 was the response variable, larval diet (rich or poor) was the fixed categorical predictor, and

138 trait I.D. was the random effect. Subsequently, we used parametric bootstrapping (1000

139 iterations, 500 permutations) to obtain uncertainty in estimated repeatability values using

140 the package "rptR" (Stoffel et al., 2017).

141 On the mean repeatability estimates for each trait, we conducted a paired Wilcoxon signed 142 rank test to compare estimates obtained via the remounting vs. remeasuring methods. We

143 then fit a linear mixed model to the mean repeatability estimates, with method

144 (remeasuring vs. remounting) as a fixed categorical predictor, mean trait size as a fixed

145 covariate, and the two-way interaction between method and trait size and Trait_ID as the

146 random factor. Conditional effect sizes reflect the variance explained by both the fixed and

147 random effects (Nakagawa \& Schielzeth, 2013) and allow us to quantify the magnitude of

148 the influence that each factor has on the dependent variable (repeatability).Therefore, we

149 also calculated the conditional effect sizes for each factor using three models that included

150 either just method, mean trait size or the interaction between them using the technique

151 outlined by Nakagawa \& Schielzeth (2013).

\section{Results}

153 Repeatability estimates obtained via remounting were lower than estimates obtained via

154 remeasuring for each of the 22 traits (Table 1) (Wilcoxon signed-rank test, $w=496, p$

$155<0.001)$. The difference between repeatability estimates obtained via remeasuring and

156 remounting methods increased as remounted repeatability decreased $(r=-0.98, t=-28.1$,

$157 p<0.001 ;$ Fig. 3). There was a significant interaction between trait size and measurement

158 methodology whereby the difference between estimates obtained via remounting vs.

159 remeasuring was greater for smaller traits (Table 2). As traits increased in size, and

160 therefore decreased in average difficulty of consistent measurement, differences between

161 the two methods decreased (Figure 4). 
162 Table 1. Estimates of trait repeatability based on remounting and remeasuring methods for

16322 morphometric traits of Telostylinus angusticollis. Traits are ordered by decreasing mean

164 size $(\mathrm{mm})$.

Repeatability (remeasured) Repeatability (remounted)

\begin{tabular}{|c|c|c|c|c|c|}
\hline Trait type & Trait & Sex & mean trait size $(\mathrm{mm})$ & {$[95 \% \mathrm{Cl}]$} & {$[95 \% \mathrm{Cl}]$} \\
\hline Somatic & FSp & male & 0.051 & $0.919[0.881,0.946]$ & $0.777[0.672,0.859]$ \\
\hline Genitalic & Ant & female & 0.063 & $0.995[0.992,0.997]$ & $0.901[0.847,0.939]$ \\
\hline Genitalic & PS1 & female & 0.073 & $0.995[0.993,0.997]$ & $0.932[0.892,0.957]$ \\
\hline Genitalic & PS2 & female & 0.075 & $0.994[0.991,0.996]$ & $0.919[0.876,0.949]$ \\
\hline Somatic & SB & male & 0.161 & $0.994[0.990,0.996]$ & $0.760[0.655,0.847]$ \\
\hline Genitalic & SP & male & 0.170 & $0.977[0.964,0.985]$ & $0.863[0.794,0.912]$ \\
\hline Genitalic & Sur_P & male & 0.177 & $0.967[0.949,0.978]$ & $0.835[0.750,0.894]$ \\
\hline Genitalic & Sur_D & male & 0.295 & $0.980[0.970,0.987]$ & $0.759[0.642,0.843]$ \\
\hline Somatic & $\mathrm{TE}$ & male & 0.378 & $0.996[0.995,0.998]$ & $0.943[0.906,0.965]$ \\
\hline Somatic & $\mathrm{PP}$ & female & 0.519 & $0.941[0.914,0.960]$ & $0.649[0.501,0.759]$ \\
\hline Somatic & PP & male & 0.659 & $0.975[0.963,0.983]$ & $0.865[0.795,0.913]$ \\
\hline Somatic & $A R$ & female & 1.050 & $0.987[0.980,0.992]$ & $0.810[0.728,0.872]$ \\
\hline Somatic & AR & male & 1.077 & $0.994[0.991,0.996]$ & $0.800[0.690,0.871]$ \\
\hline Genitalic & Apod & male & 1.091 & $0.964[0.946,0.977]$ & $0.827[0.736,0.889]$ \\
\hline Genitalic & $\mathrm{AL}$ & male & 1.136 & $0.996[0.994,0.998]$ & $0.808[0.712,0.879]$ \\
\hline Somatic & HW & female & 1.309 & $0.993[0.990,0.996]$ & $0.861[0.800,0.906]$ \\
\hline Somatic & $H W$ & male & 1.316 & $0.993[0.990,0.996]$ & $0.865[0.794,0.912]$ \\
\hline Somatic & $\mathrm{HL}$ & female & 1.705 & $0.993[0.989,0.995]$ & $0.854[0.788,0.903]$ \\
\hline Genitalic & $P R$ & male & 1.864 & $0.984[0.976,0.990]$ & $0.938[0.902,0.962]$ \\
\hline Genitalic & OVL & female & 1.946 & $0.991[0.987,0.994]$ & $0.929[0.893,0.952]$ \\
\hline Somatic & $\mathrm{HL}$ & male & 2.240 & $0.998[0.997,0.999]$ & $0.922[0.881,0.950]$ \\
\hline Somatic & $\mathrm{TL}$ & female & 2.340 & $0.994[0.991,0.996]$ & $0.927[0.889,0.953]$ \\
\hline Somatic & $\mathrm{TL}$ & male & 2.545 & $0.997[0.996,0.998]$ & $0.959[0.937,0.974]$ \\
\hline Somatic & Fta & Female & 2.589 & $0.992[0.988,0.995]$ & $0.968[0.950,0.980]$ \\
\hline Somatic & WL & Female & 3.460 & $0.999[0.999,0.999]$ & $0.990[0.984,0.994]$ \\
\hline Somatic & Fta & male & 3.583 & $0.993[0.989,0.996]$ & $0.953[0.925,0.972]$ \\
\hline Somatic & WL & male & 3.671 & $0.998[0.997,0.999]$ & $0.977[0.964,0.986]$ \\
\hline Somatic & FT & female & 3.743 & $0.992[0.988,0.995]$ & $0.959[0.939,0.973]$ \\
\hline Somatic & $\mathrm{FE}$ & female & 4.354 & $0.997[0.996,0.998]$ & $0.976[0.964,0.985]$ \\
\hline Somatic & $\mathrm{FT}$ & male & 4.877 & $0.988[0.982,0.992]$ & $0.961[0.938,0.975]$ \\
\hline Somatic & $\mathrm{FE}$ & male & 4.946 & $0.999[0.998,0.999]$ & $0.982[0.972,0.989]$ \\
\hline
\end{tabular}


165

166 Table 2. Generalized linear mixed effects model of repeatability with conditional effect

167 sizes.

\begin{tabular}{lcccccc} 
& Estimate & Std. Error & $d f$ & $F$ & $p$ & $R^{2} G L M M(c)$ \\
\hline (Intercept) & 0.980 & 0.013 & 46.146 & - & - & - \\
Mean trait size & -0.155 & 0.016 & 36.412 & 95.578 & $<0.001$ & $15.84 \%$ \\
Method & 0.004 & 0.006 & 36.528 & 18.147 & 0.497 & $59.84 \%$ \\
Method: Mean trait size & 0.032 & 0.007 & 36.412 & 21.039 & $<0.001$ & $72.66 \%$ \\
\hline
\end{tabular}

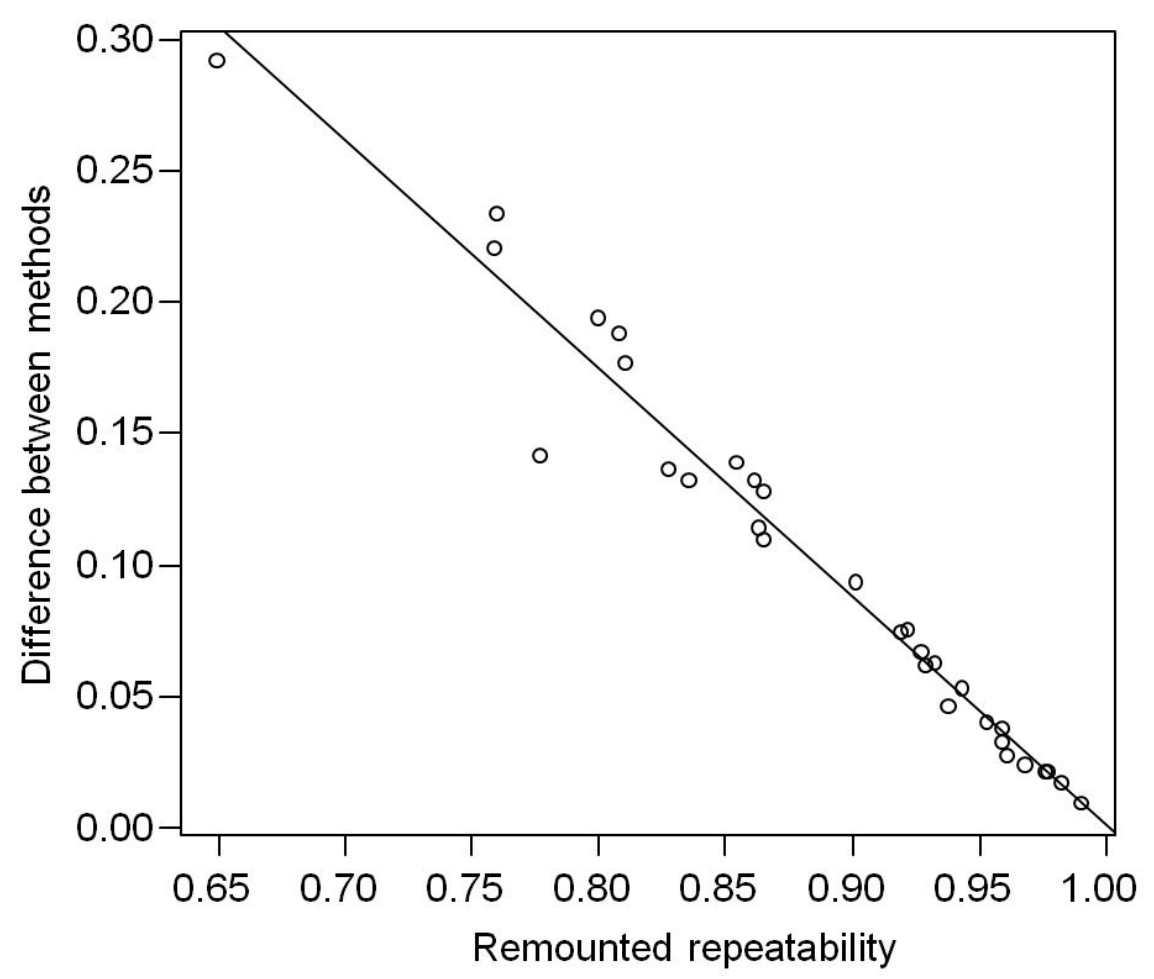

168

169 Fig. 3. The difference between repeatability estimates obtained via remeasuring and

170 remounting methods increases as remounted repeatability decreases.

171

172 


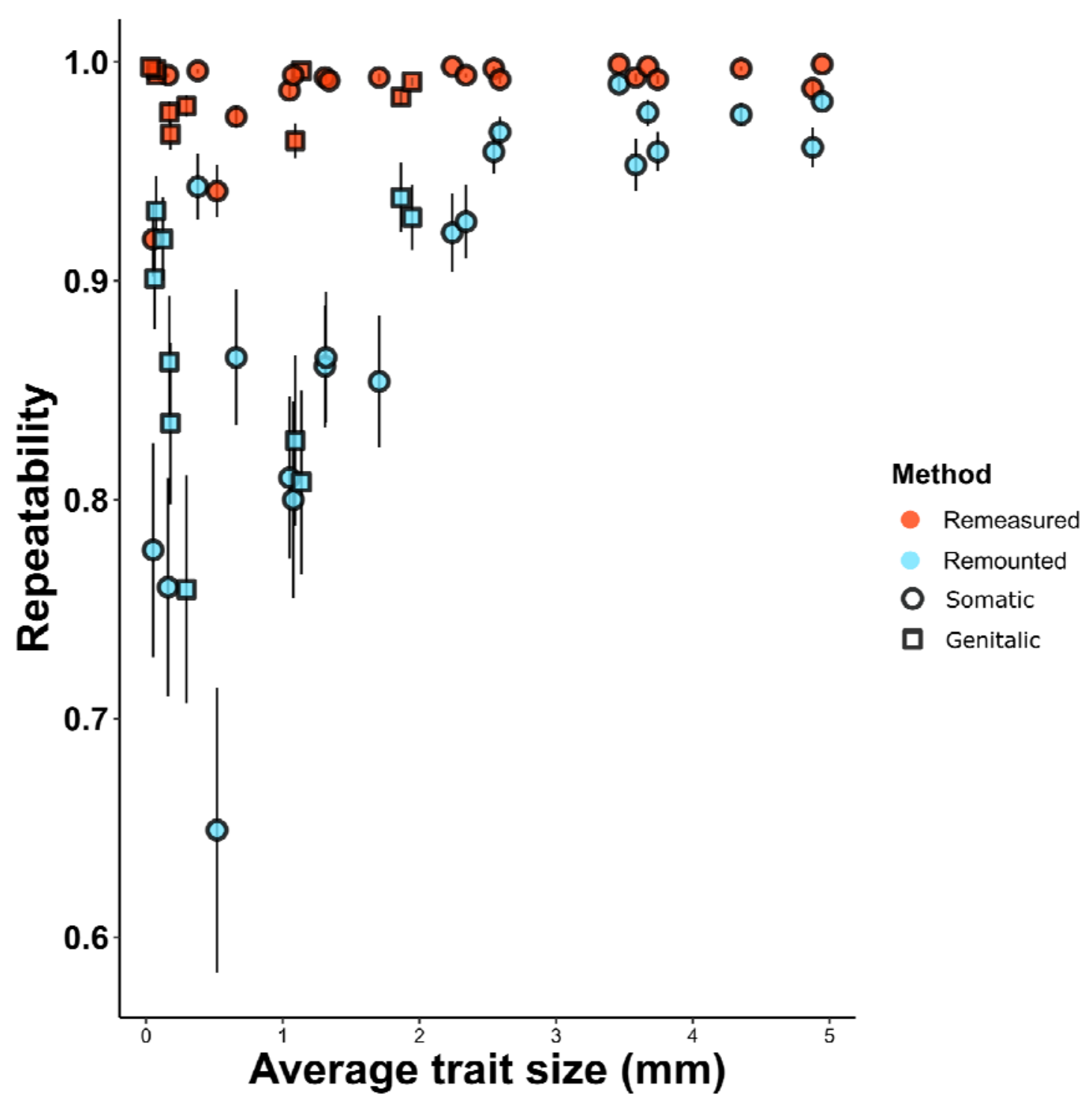

175 Fig.4. Estimates of repeatability obtained by remounting and remeasuring methods, ranked

176 by mean trait size (smallest to largest, left to right). Bars represent standard error of the

177 mean calculated from bootstrapped estimates of repeatability. 
181 It is clear from our results that both the sample-handling method and the nature of the trait

182 itself can strongly influence estimates of repeatability. For each of the 22 traits examined,

183 we found that repeatability estimates obtained by remounting samples between successive

184 measurements were smaller than repeatability estimates obtained by remeasuring a single

185 mount. Moreover, the difference between the estimates obtained via the two methods

186 increased on average as trait size decreased. Small, soft, or rounded body parts are likely to

187 be subject to greater measurement error than large, hard or flat parts, and remeasuring a

188 single mount is likely to substantially underestimate measurement error for such traits and

189 yield substantially inflated estimates of measurement repeatability. The inflation of

190 repeatability can have important consequences, potentially leading to overestimates of

191 statistical power or parameters of interest such as heritability.

192 Repeatability is often used to quantify the accuracy and consistency of phenotypic

193 measurements in evolutionary and behavioural ecology. In morphometric studies,

194 repeatabilities are often reported as a guide to the signal-to-noise ratio of various trait

195 measurements. This can be useful in gauging whether a stronger effect for a particular trait,

196 relative to other traits, might simply reflect differences among traits in measurement error

197 e.g. (Cassidy, Bath, Chenoweth, \& Bonduriansky, 2013). Repeatability estimates are also

198 sometimes used to estimate the upper bounds of narrow- and broad-sense heritability

199 (Boake, 1989; Falconer \& Mackay, 1996; Lynch \& Walsh, 1998; Dohm, 2002). Furthermore,

200 repeatabilities have been used to test predictions of condition-dependent models of sexual

201 selection. For example, Foley et al (2012) found that for cervid antler traits, repeatability

202 declines as environmental variation increases, supporting the idea that antlers serve as an

203 honest signal of individual condition. Our findings suggest that estimates of repeatability

204 obtained by remounting specimens between successive measurements are more accurate,

205 and that the importance of using this method, rather than simply remeasuring a single

206 mount or image, increases as trait measurement error increases. Interestingly, while the

207 difference between methods was small for all of the large structures in our sample (i.e.,

208 structures $>2.5 \mathrm{~mm}$ in length), the difference between methods varied considerably for the

209 smaller structures (Fig. 4). This suggests that trait size does not account fully for variation in

210 measurement error and that other factors (e.g. shape or degree of sclerotization) could also

211 be important. 
212 Although we have focused in this study on morphometric repeatability, the principles that

213 we discuss generalize to research on other types of traits for which repeatability is

214 quantified. In all studies that report repeatability, it is important to consider the major

215 sources of measurement error or variability, and how to estimate repeatability in a way that

216 captures these factors (Wilson 2018). For example, many studies estimate repeatability for

217 individual behaviour as a way to describe 'behavioural syndromes' or 'animal personalities'

218 (Sih, Bell, \& Johnson, 2004; Bell, Hankison, \& Laskowski, 2009). In such studies, rather than

219 retesting individual responses under identical conditions, it may be more relevant to test

220 responses across different types of arenas or social environments. Behaviours remeasured

221 in different contexts will probably be less repeatable (Fisher, James, Rodríguez-Muñoz, \&

222 Tregenza, 2015; Arvidsson, Adriaensen, van Dongen, De Stobbeleere, \& Matthysen, 2017),

223 but such estimates may be more meaningful if the aim is to quantify the consistency in

224 individual responses across the range of contexts where the behaviour is expressed. For

225 example, if the aim is to estimate the repeatability of a behavioural courtship signal, then it

226 may be useful to measure this signal in multiple contexts where this signal is employed.

227 Simply remeasuring the signal within the same context may yield a misleading estimate of 228 signal repeatability.

229 In conclusion, our findings suggest that remeasuring from the same mount can yield

230 strongly inflated repeatability estimates in morphometric studies, especially for traits

231 subject to large measurement error. Remounting samples between measurements is likely

232 to provide more meaningful estimates of repeatability. More broadly, the methods used to

233 estimate repeatability should capture as many important sources of measurement error or

234 variability as possible.

235 Data accessibility

236 All data will be available on the Dryad data repository.

\section{References}

238 Altaye, M., Donner, A., \& Klar, N. (2001). Inference procedures for assessing interobserver

239 agreement among multiple raters. Biometrics, 57(2), 584-588. doi:10.1111/j.0006-

$240 \quad 341 X .2001 .00584 . x$ 
241 Arvidsson, L. K., Adriaensen, F., van Dongen, S., De Stobbeleere, N., \& Matthysen, E. (2017).

242 Exploration behaviour in a different light: testing cross-context consistency of a common

243 personality trait. Animal Behaviour, 123, 151-158. doi:10.1016/j.anbehav.2016.09.005

244 Bailey, R. C., \& Byrnes, J. (1990). Society of Systematic Biologists A New , Old Method for Assessing

245 Measurement Error in Both Univariate and Multivariate Morphometric Studies. Syst. Zool.,

$24639(2), 124-130$.

247 Bates, D., Maechler, M., Bolker, B., \& Walker, S. (2015). Fitting Linear Mixed-Effects Models Using

248 Ime4. Journal of Statistical Software. Journal of Statistical Software, 67(1), 1-48.

249 Bell, A. M., Hankison, S. J., \& Laskowski, K. L. (2009). The repeatability of behaviour: a meta-analysis.

$250 \quad$ Animal Behaviour, 77(4), 771-783. doi:10.1016/j.anbehav.2008.12.022

251 Bertin, A., \& Fairbairn, D. J. (2007). The form of sexual selection on male genitalia cannot be inferred 252 from within-population variance and allometry - A case study in Aquarius remigis. Evolution, 253 61(4), 825-837. doi:10.1111/j.1558-5646.2007.00074.x

254 Blanckenhorn, W. U., Kraushaar, U. R. S., Teuschl, Y., \& Reim, C. (2004). Sexual selection on 255 morphological and physiological traits and fluctuating asymmetry in the black scavenger fly 256 Sepsis cynipsea. Journal of Evolutionary Biology, 17(3), 629-641. doi:10.1111/j.1420-

$257 \quad 9101.2004 .00693 . x$

258 Boake, C. R. B. (1989). Repeatability: Its role in evolutionary studies of mating behavior. Evolutionary Ecology, 3(2), 173-182. doi:10.1007/BF02270919

Bonduriansky, R. (2007). The evolution of condition dependent sexual dimorphism. The American Naturalist, 169, 9-19.

Bonduriansky, R. (2006). Convergent evolution of sexual shape dimorphism in Diptera. Journal of Morphology, 267, 602-611.

Bonduriansky, Russell, Mallet, M. A., Arbuthnott, D., Pawlowsky-Glahn, V., Egozcue, J. J., \& Rundle, H. D. (2015). Differential effects of genetic vs. environmental quality in Drosophila melanogaster suggest multiple forms of condition dependence. Ecology Letters, 18(4), 317326. doi:10.1111/ele.12412 morphological diversification: evolution of reaction norms and static allometries in neriid flies. Evolution, 68, 368-383. 
271 Cayetano, L., \& Bonduriansky, R. (2015). Condition dependence of male and female genital 272 structures in the seed beetle Callosobruchus maculatus (Coleoptera: Bruchidae). Journal of 273 Evolutionary Biology, 28(7), 1364-1372. doi:10.1111/jeb.12659

274 Dohm, M. R. (2002). Repeatability estimates do not always set an upper limit to heritability.

275 Functional Ecology, 16(2), 273-280. doi:10.1046/j.1365-2435.2002.00621.x

276 Falconer, D. S., \& Mackay, C. (1996). Introduction to quantitative genetics (4th ed.).

277 Fisher, D. N., James, A., Rodríguez-Muñoz, R., \& Tregenza, T. (2015). Behaviour in captivity predicts 278 some aspects of natural behaviour, but not others, in a wild cricket population. Proceedings of 279 the Royal Society B: Biological Sciences, 282(1809). doi:10.1098/rspb.2015.0708

280 Foley, A. M., DeYoung, R. W., Lukefahr, S. D., Lewis, J. S., Hewitt, D. G., Hellickson, M. W., ... 281 DeYoung, C. A. (2012). Repeatability of antler characteristics in mature white-tailed deer in 282 South Texas: consequences of environmental effects. Journal of Mammalogy, 93(4), 1149283 1157. doi:10.1644/11-mamm-a-183.2

284 Ghosh, H., \& Das, A. (2003). Optimal diallel cross designs for estimation of heritability. Journal of Statistical Planning and Inference, 116(1), 185-196. doi:10.1016/S0378-3758(02)00180-5

Hosken, D. J., Minder, A. M., \& Ward, P. I. (2005). Male genital allometry in Scathophagidae (Diptera). Evolutionary Ecology, 19(5), 501-515. doi:10.1007/s10682-005-1023-z

Kuznetsova, A., Brockhoff, P. B., \& Christensen, R. H. B. (2017). \{lmerTest\} Package: Tests in Linear Mixed Effects Models. Journal of Statistical Software, 82(13), 1-26. doi:10.18637/jss.v082.i13 104, 116-121.

Lynch, M., \& Walsh, B. (1998). Genetics and Analysis of Quantitative Traits. Sinauer, Massachussetts. generalized linear mixed-effects models. Methods in Ecology and Evolution, 4(2), 133-142. doi:10.1111/j.2041-210x.2012.00261.x

R Core Team. (2019). R: A Language and Environment for Statistical Computing. Vienna, Austria. 
Ecology, 27, 1134-1144.

301 Shoukri, M. M., \& Donner, A. (2001). Efficiency considerations in the analysis of inter-observer

302 agreement. Biostatistics, 2(3), 323-336. doi:10.1093/biostatistics/2.3.323

303 Sih, A., Bell, A., \& Johnson, J. C. (2004). Behavioral syndromes: An ecological and evolutionary

$304 \quad$ overview. Trends in Ecology and Evolution, 19(7), 372-378. doi:10.1016/j.tree.2004.04.009

305 Sokal, R. R., \& Rohlf, F. J. (1995). Biometry the Principles and Practice of Statistics in Biological

306 Research (3rd ed.). New York: Freeman and Company.

307 Stoffel, M. A., Nakagawa, S., \& Schielzeth, H. (2017). rptR: repeatability estimation and variance 308 decomposition by generalized linear mixed-effects models. Methods in Ecology and Evolution,

309 8. doi:10.1111/2041-210X.12797

310 Wilson, A. J. (2018). How should we interpret estimates of individual repeatability? Evolution Letters,

$3112(1), 4-8$. doi:10.1002/evl3.40

312 Wolak, M. E., Fairbairn, D. J., \& Paulsen, Y. R. (2012). Guidelines for estimating repeatability.

313 Methods in Ecology and Evolution, 3(1), 129-137. doi:10.1111/j.2041-210X.2011.00125.x

314 Yezerinac, S. M., Lougheed, S. C., \& Handford, P. (1992). Measurement Error and Morphometric

315 Studies]: Statistical Power and Observer Experience. Syst. Biol., 41(4), 471-482. 